\title{
A study of the antioxidant capacity of oak wood used in wine ageing and the correlation with polyphenol composition
}

Article

Accepted Version

Alañón, M.E., Castro-Vázquez, L., Díaz-Maroto, M.C., Gordon, M. H. and Pérez-Coello, M.S. (2011) A study of the antioxidant capacity of oak wood used in wine ageing and the correlation with polyphenol composition. Food Chemistry, 128 (4). pp. 997-1002. ISSN 0308-8146 doi:

https://doi.org/10.1016/j.foodchem.2011.04.005 Available at https://centaur.reading.ac.uk/20442/

It is advisable to refer to the publisher's version if you intend to cite from the work. See Guidance on citing.

To link to this article DOI: http://dx.doi.org/10.1016/j.foodchem.2011.04.005

Publisher: Elsevier

All outputs in CentAUR are protected by Intellectual Property Rights law, including copyright law. Copyright and IPR is retained by the creators or other copyright holders. Terms and conditions for use of this material are defined in the End User Agreement. 


\section{CentAUR}

Central Archive at the University of Reading

Reading's research outputs online 
$18 *$ Corresponding author:

19 Tlf.: [34] 926295300 Ext. 3425

20 Fax: [34] 926295318

21 e-mail: mariaelena.alanon@uclm.es

\section{COMPOSITION}

\section{M.E. Alañón ${ }^{1 *}$, L. Castro-Vázquez ${ }^{1}$, M.C. Díaz-Maroto², M.H. Gordon ${ }^{3}$, M.S.} Pérez-Coello ${ }^{1}$

${ }^{1}$ Área de Tecnología de los Alimentos, Facultad de Ciencias Químicas, Universidad de Castilla-La Mancha, Av. Camilo José Cela 10, 13071, Ciudad Real, Spain

${ }^{2}$ IRICA (Instituto Regional de Investigación Científica Aplicada), Universidad de Castilla La-Mancha, Campus Universitario, s/n, 13071 Ciudad Real, Spain

${ }^{3}$ Department of Food and Nutritional Sciences, University of Reading, Whiteknights PO Box 226, Reading, RG6 6AP, United Kingdom 


\section{Abstract}

28 The antioxidant capacity of oak wood used in the ageing of wine was studied by four

29 different methods: measurement of scavenging capacity against a given radical (ABTS,

30 DPPH), oxygen radical absorbance capacity (ORAC) and the ferric reducing antioxidant

31 power (FRAP). Although, the four methods tested gave comparable results for the

32 antioxidant capacity measured in oak wood extracts, the ORAC method gave results

33 with some differences from the other methods. Non-toasted oak wood samples

34 displayed more antioxidant power than toasted ones due to differences in the

35 polyphenol compositon. A correlation analysis revealed that ellagitannins were the

36 compounds mainly responsible for the antioxidant capacity of oak wood. Some phenolic

37 acids, mainly gallic acid, also showed a significant correlation with antioxidant 38 capacity.

40 Keywords: Antioxidant capacity, oak wood, wine ageing, phenolic compounds. 


\section{Introduction}

52

In recent decades increasing interest in natural antioxidant present in the diet has

developed among consumers and the scientific community. Natural antioxidants seem

to play a very important role in reducing the concentration of free radicals, which are harmful and highly reactive intermediates constantly produced due to numerous biological reactions. Antioxidants prevent the oxidation process thanks to their capacity for capturing, de-activating or repairing the damage caused by free radicals which are implicated in the development of multiple diseases.

60

Epidemiological studies have indicated that frequent intake of natural dietary antioxidants is associated with a lower risk of cardiovascular disease and cancer

63 (Renaud, Guegue, Schenker, \& d’Houtaud, 1998; Kaur \& Dapoor, 2001; Record, Dreosti, \& Mclnerney, 2001). Fruits, vegetables and all the foods and drinks derived from these commodities are the main source of natural antioxidants due to their high content of polyphenols. Wine has been one the most studied beverages due to its verified antioxidant potential and health benefits attributable to its high content of polyphenols, which are present in solution with good bioavailability (Renaud et al., 1998; Tomera, 1999).

The ageing process is a common technological procedure used in winemaking which seems to contribute to an increase in the antioxidant capacity of wines (Larrauri,

73 Sánchez-Moreno, Rupérez, \& Saura-Calixto, 1999; Canas, Casanova, \& Belchior, 2008;

74 Alonso, Castro, Rodríguez, Guillén, \& Barroso, 2004) This is due to the important 
amount of polyphenols which is extracted from the oak wood during contact with wine during the ageing stage.

77

difficult task because wines are complicated mixtures rich in polyphenols and oenological practices including sulphur dioxide addition (Manzocco, Mastrocloa, \& Nicoli, 1999), skin contact (Fuhrman, Volkova, Suraski, \& Aviram, 2001), carbonic maceration (Pellegrini, Simonetti, Gardana, Brema, Brighenti, \& Pietta, 2000), vinification conditions (Burns, Gardner, Matthews, Duthie, Lean, \& Crozier, 2001) and microoxygenation (Rivero-Pérez, Gónzalez-Sanjosé, Muñiz, \& Pérez-Magariño, 2008), which can all influence the antioxidant capacity.

Therefore, despite the demonstrated increase in antioxidant capacity of aged wines, there are no previous reports of the antioxidant capacity acquired from oak wood. The toasting process is a crucial practice used in cooperage that causes deep changes in oak wood chemical composition (Hale, McCafferty, Larmie, Newton, \& Swan, 1999) which could affect the antioxidant capacity of oak wood. Therefore, we were interested in the study of antioxidant capacity taken up from oak wood used in cooperage for aging wine and how the toasting process affects it.

Despite many analytical methods being available for assessing antioxidant capacity in vitro, there are no approved standardised methods. Sometimes, this diversity of methodologies used to evaluate natural antioxidants has led to widely conflicting results that are extremely difficult to interpret (Frankel \& Meyer, 2000). For instance, Ou et al (Ou, Huang, Hampsch-Woodill, Glanagan, \& Deemer, 2002) reported no 
100 correlation of antioxidant activity between values determined by the FRAP (ferric

101 reducing antioxidant power) and ORAC (oxygen radical absorption capacity)

102 techniques among most of the 927 freeze-dried vegetable samples. They concluded that

103 the ORAC method is chemically more relevant to the activity of chain-breaking

104 antioxidants but only measures activity against peroxyl radicals. In contrast the FRAP

105 assay estimates only the Fe III reducing activity, which is not necessarily the

106 antioxidant activity. Furthermore the FRAP assay has some drawbacks due to

107 interference, reaction kinetics, etc. Fernandez-Pachon, Villaño, García-Parilla, \&

108 Troncoso (2004) determined that the antioxidant activity evaluated as the capacity to

109 quench radicals assessed by means of the ABTS (3-ethyl-benzothiazoline-6-sulfonic

110 acid) and DPPH (diphenyl-1-picrylhydrazyl) methods is 10 or 15-times higher for red

111 wines than for white ones. However, according to the ORAC assay, red wines are only

112 five-times more active than white wines.

114 These differences are mainly attributable to the different chemistry principles

115 underlying these methods. Other factors such as matrix type, hydrophilic/lipophilic

116 character of compounds and heterogeneity of substrates can also influence the results

117 obtained. Therefore, a valid evaluation of antioxidant capacity requires the use of

118 several methods with different mechanisms for inhibiting oxidation (Frankel et al., 119 2000).

The aim of this work was to gain an insight into the validity of existing methodologies for the evaluation of the antioxidant characteristics of oak wood.

123 Selected methods include the measurement of scavenging capacity against a given

124 radical (ABTS, DPPH), the oxygen radical absorbance capacity (ORAC) and the ferric 
125 reducing antioxidant power (FRAP). They have been applied to aqueous alchoholic

126 extracts of samples of oak woods prepared to simulate the wine aging process. The

127 antioxidant capacity conferred by two types of oak woods, toasted and non toasted, of

128 different provenance was evaluated. In addition, the relationship between antioxidant

129 capacity and phenolic composition (phenolic acids and ellagitannins) has also been

130 considered in order to evaluate the contribution of each phenolic compound to

131 antioxidant capacity.

\section{2. Materials and methods}

\subsection{Samples}

Shavings from oak wood samples (sized $2 \mathrm{~cm} \times 1 \mathrm{~cm} \times 0.1 \mathrm{~cm}$ ) collected with supplied by the cooperage Magreñan S.L. (La Rioja, Spain). Samples were naturally

140 seasoned in the open air and one section of each non-toasted oak wood sample, was

141 submitted to thermal treatment. Toasted samples received a medium intensity toasting 142 (45-50 min) with the temperature of the wood surface being: $160-170{ }^{\circ} \mathrm{C}$.

\subsection{Extractions}

In order to investigate the extraction of oak wood compounds from wines during

147 a simulated aging process, fourteen grams of shavings of each wood sample were

148 soaked in a litre of synthetic wine model solution (12\% ethanol v/v adjusted to $\mathrm{pH} 3.5$

149 with tartaric acid). (Pérez-Coello, Sanchéz, García, Gonzalez-Viñas, Sanz, \& Cabezudo, 
150 2000). The solutions, prepared in duplicate, were shaken daily and after three weeks,

151 filtered and kept refrigerated until their analyses.

\subsection{Total phenolic index}

The total phenol content of extracts was determined according to the Folin-

155 Ciocalteu procedure (Singleton, \& Rossi, 1965). Deionised water (1.8 mL) was added to

$1560.2 \mathrm{~mL}$ of each extract. Folin-Ciocalteu reagent $(0.2 \mathrm{~mL})$ was then added and tubes 157 were shaken vigorously. After 3 minutes, $0.4 \mathrm{~mL}$ sodium carbonate solution $(35 \% \mathrm{w} / \mathrm{v})$

158 was added, along with $1.4 \mathrm{~mL}$ of deionised water. Samples were well mixed and left in

159 the dark for 1 hour. The absorbance was measured at $725 \mathrm{~nm}$ using a UV-vis

160 spectrophotometer (Lambda 5, Perkin-Elmer, Seer Green, UK)) and the results were 161 expressed in gallic acid equivalents, GAE, using a gallic acid standard curve (0-0.2 $162 \mathrm{mg} / \mathrm{mL}$ ). Extracts were further diluted if the absorbance value measured was above the 163 linear range of the standard curve.

2.4. Antioxidant capacity determination

166 2.4.1. DPPH assay

167

The DPPH assay was carried out according to the method of Brand-Williams,

169 Cuvelire, \& Berset, (1995) where 1,1-diphenyl-2-picrylhydrazyl radical was used as a

170 stable radical. One hundred microliters of different dilutions of extracts were added to

$1713.9 \mathrm{~mL}$ of a $0.06 \mathrm{mM}$ methanol DPPH radical solution. Methanol was used to adjust the

172 zero and the decrease in absorbance was measured at $515 \mathrm{~nm}$ every minute for 25

173 minutes in a UV-vis spectrophotometer (He $\lambda$ ios, Thermo Spectronic, Cambridge, UK).

174 Only values between 20 and $80 \%$ of the initial absorbance of the radical DPPH were 
175 taken into consideration. Concentrations were calculated from a calibration curve in the 176 range between 0.1 and $0.8 \mathrm{mM}$ Trolox. Results were expressed in $\mu \mathrm{M}$ Trolox 177 equivalents per milligram of oak wood. The method used was the ABTS++ (radical cation) decolorisation assay (Re, 182 Pellegrini, Proteggente, Pannala, Yang, \& Rice-Evans, 1999). The assay is based on the ability of an antioxidant compound to quench the $\mathrm{ABTS}^{++}$relative to that of a reference antioxidant such as Trolox. A stock solution of $\mathrm{ABTS}^{+}$radical cation was prepared by mixing ABTS solution and potassium persulfate solution at $7 \mathrm{mM}$ and $2.45 \mathrm{mM}$ final concentration respectively. The mixture was maintained in the dark at room temperature for 12-16 hours before use. The working $\mathrm{ABTS}^{++}$solution was produced by dilution in ethanol $(1: 90 \mathrm{v} / \mathrm{v})$ of the stock solution to achieve an absorbance value of $0.7( \pm 0.02)$ at $734 \mathrm{~nm}$. An aliquot of $20 \mu \mathrm{L}$ of diluted extract was added to ABTS $^{++}$working solution

190 (2 mL). For the blank and standard curve, $20 \mu \mathrm{L}$ of ethanol or Trolox solution was used

191 respectively. Absorbance was measured by means of a UV-vis spectrophotometer 192 (Perkin Elmer Lambda 5) at $734 \mathrm{~nm}$ immediately after addition and rapid mixing $\left(\mathrm{A}_{\mathrm{t}=0}\right)$ and then every minute for 5 minutes. Readings at $t=0 \min \left(A_{t=0}\right)$ and $t=5 \min \left(A_{t=5}\right)$ of reaction were used to calculate the percentage inhibition value for each extract.

A standard reference curve was constructed by plotting \% inhibition value against Trolox concentration $(0-15 \mu \mathrm{M})$. The radical-scavenging capacity of extracts was quantified as $\mu \mathrm{mol}$ of Trolox equivalent per milligram of oak wood. 
The FRAP assay was performed as previously described by Benzie and Strain

203 (Benzie, \& Strain, 1999) with some modifications. This spectrophotometric assay 204 measures the ferric reducing ability of antioxidants. The experiment was conducted at $20537^{\circ} \mathrm{C}$ and $\mathrm{pH}$ 3.6. In the FRAP assay, reductants ("antioxidants") present in the extract 206 reduce $\mathrm{Fe}$ (III)-tripyridyltriazine complex to the blue ferrous form, with an absorption 207 maximum at $593 \mathrm{~nm}$. The assay was performed by means of automated microplate 208 reader (Tecan GENios Pro, (Tecan Ltd, Dorset, UK)) with 96-well plates. Reagents 209 included $300 \mathrm{mM}$ acetate buffer $\mathrm{pH}$ 3.6; 40mM hydrochloric acid; $10 \mathrm{mM}$ TPTZ 210 solution and $20 \mathrm{mM}$ ferric chloride solution. The working FRAP reagent was prepared 211 fresh on the day of analysis by mixing acetate buffer, TPTZ solution and ferric chloride 212 solutions in the ratio 10:1:1 and the mixture was incubated at $37^{\circ} \mathrm{C}$. Diluted extract $(30$ $213 \mu \mathrm{L})$ and pre-warmed FRAP reagent $(225 \mu \mathrm{L})$ were put into each well. The absorbance at 214 time zero and after 4 min was recorded at $593 \mathrm{~nm}$. The calculated difference in 215 absorbance is proportional to the ferric reducing/antioxidant power of the extract. For 216 quantification, a calibration curve of Trolox was prepared with dilutions from $0 \mu \mathrm{M}$ to $217750 \mu \mathrm{M}$. The final results were expressed as $\mu$ mol of Trolox equivalent per milligram of 218 oak wood.

\subsubsection{ORAC assay}

222 The method of the ORAC assay was adapted from Cao, \& Prior (1999). The assay was 223 performed with an automated microplate reader and 96-well plates. The perimeter wells 224 were not used for samples, but they were filled with $250 \mu 1$ of water to ensure all sample 
wells were surrounded by full wells. Diluted extract $(25 \mu \mathrm{l})$ was pipetted into each well

226 and then fluorescein working solution $(96 \mathrm{nM}, 150 \mu \mathrm{l})$ in phosphate buffer, $\mathrm{pH} 7.4$ and

$22737^{\circ} \mathrm{C}$, wwas added to each sample. The plate was placed in a Genios spectrophotometer

228 (Tecan Ltd, Dorset, UK) and incubated for 10 minutes at $37^{\circ} \mathrm{C}$. The initial fluorescence

229 was recorded at an excitation wavelength of $485 \mathrm{~nm}$ and an emission wavelength of 535

230 nm. 2,2'-Azobis(2-amidopropane)di-hydrochloride (AAPH, $153 \mathrm{mM}, 75 \mu \mathrm{l}$ ) was then

231 added to each sample well and the fluorescence was measured immediately and every 5

232 minutes thereafter for 150 minutes. The procedure was repeated using solutions of

233 Trolox in the range $0 \mu \mathrm{M}$ to $100 \mu \mathrm{M}$ to prepare the calibration curve. The ORAC value

234 for each extract was calculated using a regression equation relating Trolox

235 concentration to the net area under the fluorescence decay curve (AUC). Results are

236 expressed as $\mu$ mols of Trolox equivalents per milligram of oak wood.

237

2.5. HPLC-DAD-ESI-MS ${ }^{n}$ Analysis of Phenolic Acids.

Standards of $p$-coumaric acid, gallic acid, ferulic acid, caffeic acid, vanillic acid, protocatechuic acid and protocatechuic aldehyde were acquired from Sigma (St. Louis, MO, USA) and sinapic acid, syringaldehyde, coniferaldehyde, sinapaldehyde, 4hydroxybenzoic acid, vanillin were provided by Fluka (Buchs, Switzerland).

Identification and quantification of low molecular weight phenolic compounds in aqueous alcoholic extracts was achieved by HPLC analysis using an Agilent 1100 series system (Agilent, Waldbronn, Germany), equipped with a DAD photodiode detector (G1315B) and a LC/MSD Trap VL (G2445C VL) electrospray ionisation mass spectrometry $\left(\mathrm{ESI} / \mathrm{MS}^{\mathrm{n}}\right)$ system, both coupled to an Agilent Chem Station (version 
250 B.01.03) for data processing. The aqueous alcoholic extracts $(50 \mu 1)$, after filtration 251 (0.20 $\mu \mathrm{m}$, polyester membrane, Chromafil PET 20/25, Machery-Nagel, Düren, 252 Germany) were injected, in duplicate, onto a reversed-phase column Zorbax Eclipse 253 XDB-C18 (4.6 x $250 \mathrm{~mm} ; 5 \mu \mathrm{m}$ particle; Agilent), with temperature control at $40{ }^{\circ} \mathrm{C}$. 254 The solvents were water/formic acid (990:10 v/v) as solvent A; and MeOH/formic acid $255(990: 10 \mathrm{v} / \mathrm{v})$ as solvent $\mathrm{B}$. The flow rate was $0.70 \mathrm{~mL} \mathrm{~min}^{-1}$. The linear gradient for 256 solvent B was as follows: $0 \mathrm{~min}, 5 \% ; 15 \mathrm{~min}, 35 \%$; $30 \mathrm{~min}, 43 \% ; 32 \mathrm{~min}, 100 \%$; 40 $257 \min , 5 \%$.

Components were quantified using the DAD chromatograms obtained at $280 \mathrm{~nm}$

260 with external standard calibration curves. The identity of each compound was established by comparing the retention time, UV-Vis spectra and mass spectra of the peaks in every sample with those previously obtained by injection of standards. For identification, ESI-MS ${ }^{n}$ was used, setting the following parameters: positive ion mode; dry gas, $\mathrm{N}_{2}, 11 \mathrm{~mL} \min ^{-1}$; drying temperature, $350{ }^{\circ} \mathrm{C}$; nebulizer, $65 \mathrm{psi}$; capillary, $2500 \mathrm{~V}$; capillary exit offset, $70 \mathrm{~V}$; skimmer 1, $20 \mathrm{~V}$; skimmer 2, $6 \mathrm{~V}$; and scan range, $50-1200 \mathrm{~m} / \mathrm{z}$.

Ellagitannins were analysed with a 500B HPLC chromatograph (Konik Instrument, Spain) with a 7176-LC Rheodyne injection valve connected to a Konik UV-

270 Vis model 206 PHD diode-array detector. Components were detected at $325 \mathrm{~nm}$. The 271 column used was a reverse-phase C18 LiChrospher $^{\circledR} 100$ (Merck, Darmstadt) with 272 dimensions of $250 \times 4 \mathrm{~mm}$ and a particle size of $5 \mu \mathrm{m}$. Extracts $(50 \mu \mathrm{L})$ were injected 273 onto the HPLC system. The elution conditions were as follow: the flow rate was $1 \mathrm{~mL}$ $274 \min ^{-1}$ and the temperature $25^{\circ} \mathrm{C}$. Two solvents were used for elution: $\mathrm{A}: \mathrm{MeOH} / \mathrm{H}_{3} \mathrm{PO}_{4}$ 
275 (999:1 v/v) and $\mathrm{B}: \mathrm{H}_{2} \mathrm{O} / \mathrm{H}_{3} \mathrm{PO}_{4}(999: 1 \mathrm{v} / \mathrm{v})$. The gradient was: 0-8 \% $\mathrm{A}$ in 0-70 min, 8$27650 \%$ A in 70-80 min, 50-100 \% A in 80-90 min (Jordão, Ricardo, \& Laureano, 2007).

277 Quantitative results are expressed in $\mathrm{mg} \mathrm{g}^{-1}$ ellagic acid equivalents. Due to the lack of 278 commercial standards, chromatographic peaks were identified by comparing the 279 retention time and elution order with data described in the literature (Viriot, Scalbert, 280 Herve du Penhoat, Moutounet, 1994; Fernández de Simón, Cadahía, Conde, \& García281 Vallejo, 1999), and by their spectral mass obtained by the HPLC-DAD-ESI-MS ${ }^{\mathrm{n}}$ 282 instrument previously described.

\subsection{Statistical analysis}

Pearson's correlation coefficient, Student's t-test and Student-Newman-Keults test were applied to data in order to identify statistically significant differences in phenol composition and antioxidant capacity among non-toasted and toasted oak wood samples. All statistical analysis was done using SPSS v. 17.0.

\section{Results and discussions}

\subsection{Antioxidant capacity and total phenol index}

Antioxidant capacity results expressed as $\mu \mathrm{mol}$ of Trolox equivalents per

295 milligram determined by several methods for different oak wood samples under study

296 are shown in Table 1. Antioxidant capacity determined by means of different assays, 297 DPPH, FRAP, ORAC and ABTS were measured four times to test the reproducibility of 298 the assays. In all determinations, the standard deviation (sd) was lower than 0.08 . The 
magnitude of the antioxidant capacity values depended on the method employed as a

300 consequence of different conditions, reaction mechanism and end-points considered.

Non-toasted oak wood extracts showed higher antioxidant capacity values than

303 those found for toasted oak wood extracts. This fact indicates that the toasting process

304 reduces the antioxidant capacity of oak wood.

305

306 Significant differences were found between toasted and non-toasted samples in

307 the total phenol index and antioxidant capacity values estimated by DPPH, FRAP and

308 ABTS assays. However this differentiation between the types of oak wood was less

309 noticeable when the ORAC method was used. Indeed, significant differences in the

310 antioxidant capacity values assessed by the ORAC assay for toasted and non-toasted

311 oak woods were not found according to the Student-Knewman-Keuls test (Table 1).

312 Similar behaviour also was observed by Fernández-Pachón et al. (2004) who obtained

313 lower differentiation between antioxidant capacity of red and white wines when the

314 ORAC method was employed.

The correlation between the antioxidant capacity values assessed by the DDPH,

317 FRAP, ORAC and ABTS assays was investigated (Table 2). The correlations between

318 the assays studied were highly positively $(0.69<\mathrm{r}<0.97, \mathrm{P}<0.01)$, indicating that the

319 four assays provided comparable values when they were used for estimating the

320 antioxidant capacity of oak wood. A high correlation between these techniques was also

321 found by other authors in sorghum and its products and in guava fruit extracts (Awika,

322 Rooney, Wu, Prior, \& Cisneros-Zevallos, 2003; Thaipong, Boonprakob, Crosby,

323 Cisneros-Zeballos, \& Hawkins-Byrne, 2006). However, the lowest correlation 
324 coefficients were found for the ORAC assay. This fact can be attributable to the

325 different chemistry principle upon this method is based. The DPPH, FRAP and ABTS

326 methods are based on single electron transfer (SET) reaction. In these methods

327 antioxidants are oxidized by oxidants, such as a metal (Fe III) or a radical (DPPH or

$328 \mathrm{ABTS}^{+\cdot}$ ). As a result, a single electron is transferred from the antioxidant molecule to

329 the oxidant. In contrast, the ORAC assay is based on a hydrogen atom transfer (HAT)

330 reaction after a peroxyl radical ROO has been generated in which this radical abstracts

331 a hydrogen atom from the antioxidant compounds. Better correlations were found

332 between assays based on the same chemistry principle. Furthermore, the ORAC assay

333 only measures the activity of chain-breaking antioxidants against peroxyl radicals.

334 Therefore a relative difference in the ability of antioxidant compounds of oak wood in

335 the extracts to quench peroxyl radicals and to reduce $\mathrm{DPPH}, \mathrm{ABTS}^{+}$, and Fe (III) was 336 observed.

Table 1 also shows the total phenol index of the oak wood aqueous alcoholic extracts. The differences between total phenol content were consistent with those found

340 in the antioxidant capacity values. Therefore, the correlation with values from the 341 antioxidant assays tested were also evaluated (Table 2). The total phenol index, TPI, 342 showed a highly positive correlation with antioxidant capacity determined by all assays $343(0.72<\mathrm{r}<0.97, \mathrm{P}<0.01)$, which indicates that TPI is an important contributor to 344 antioxidant capacity in oak wood extracts. 
Table 3 shows the content of low molecular weight phenols in oak wood aqueous alcoholic extracts. It was evident that the toasting process caused major changes to the polyphenol composition of the oak wood samples studied. An increase in

351 the phenolic aldehydes includingprotocatechuic aldehyde, vanillin, syringaldehyde, coniferaldehyde and sinapaldehyde and some phenolic acids like vanillic, ferrulic and sinapic acids was observed. The increase of these compounds is attributable to the thermal degradation of lignin in wood, which was suffered during the toasting process (Hale et al., 1999). However, the content of $p$-coumaric acid was not significantly affected by the toasting process.

Meanwhile, an increase in the ellagic acid content of toasted samples was also observed. This change was due to the release of this compound by ellagitannins during their thermal degradation (Viriot, Scalbert, Lapierre, \& Moutounet, 1993).On the other hand, other phenolic compounds, such as gallic, protocatechuic and caffeic acids and scopoletin, were sensitive to thermal degradation causing a significant decrease in their content in toasted samples.

Others compounds studied include ellagitanins which constitute a complex class of polyphenols characterized by one or more hexahydroxydiphenoyl (HHDP) moieties esterified to a sugar, usually glucose. Table 4 shows the ellagitannin content of the oak wood samples studied. Four C-glucosidic ellagitannins monomers were detected

369 (vescalagin, castalagin, grandin, and roburin E) and also ellagitannin dimers (roburins 370 A-D). Among the ellagitannins detected, the ellagitannin monomers (gradinin, 371 vescalagina, roburin $\mathrm{E}$ and castalagin) were found in higher concentrations. Castalagin 372 was the main ellagitannin found in all samples. The toasting process had a great 
373 influence on the ellagitannin composition of oak wood, since these compounds tended

374 to decrease due to thermal degradation during the toasting process. In all cases, toasted 375 oak samples showed significantly lower ellagitannins concentrations than non-toasted 376 samples.

3.3. Correlation between antioxidant capacity and phenolic composition of oak wood.

In order to determine the contribution of individual phenolic compounds to the antioxidant capacity, the correlation between the antioxidant capacity estimated by the four methods and the concentration all the phenolic compounds detected was investigated (Table 5). The results obtained by the different methods were in good agreement. No correlation was found between the antioxidant capacity and the concentration of phenolic aldehydes (protocatecaldehyde, vanillin, coniferaldehyde and sinapaldehyde). Therefore, it can be concluded that these types of compounds do not make a amjor contribution to the antioxidant capacity of oak wood.

A significant correlation was found between antioxidant capacity and some phenolic acids including gallic acid, protocatechuic acid, caffeic acid and $p$-coumaric acid. This shows that these compounds can make a major contribution to the overall antioxidant power of oak wood. The high antioxidant activity of gallic acid has been

393 demonstrated by others authors (Canas et al., 2008; Bakkalbase, Mente, \& Artik, 2009).

394 Its antioxidant properties are a consequence of the three free phenolic hydroxyl groups 395 per molecule. However, no strong correlation between the rest of the phenolic acids and antioxidant capacity was found. 
399 concentration of all ellagitannins studied $(0.970<\mathrm{r}<0.724)$. Hence, ellagitannins, high

400 molecular weight polyphenols, are the compounds mainly responsible for the

401 antioxidant capacity of oak wood samples. This finding is consistent with reports by

402 others authors who analysed ellagitannins in different matrices (Bakkalbase et al., 2009;

403 Quideau, 2009). This activity is due to the structure of ellagitannins which is

404 characterized by the presence of several ortho hydroxyl substituents which exhibit a

405 higher ability to donate a hydrogen atom and to support the unpaired electron as

406 compared to low molecular weight phenolic compounds. Among the ellagitannins found

407 in oak wood samples, castalagin showed the highest correlation coefficient so it seems

408 to be the major contributor to antioxidant capacity, although further researches are

409 necessary to confirm this hypothesis.

410

\section{4. Conclusion}

412

On the basis of our results, it is concluded that the four methods tested (DPPH,

414 FRAP, ORAC and ABTS) gave comparable results for the antioxidant capacity of oak

415 wood extracts. However, results from the ORAC method were poorest in correlating

416 with the other methods. Furthermore the ORAC assay did not detect significant

417 differences in antioxidant capacity values between toasted and non-toasted oak wood

418 samples like the rest of the methods did.

This fact is attributed to the principle underlying the ORAC assay, which only measures the activity of chain-breaking antioxidants against peroxyl radicals. 
422 Furthermore, the ORAC assay is based on a different mechanism compared with the

423 rest of the methods.

Therefore, due to different reactive oxygen species and differences in reaction mechanisms, selecting a single method for antioxidant capacity evaluation is a rather

427 difficult task. The use of just one antioxidant capacity assay is oversimplified and thus

428 inappropriate. For that reason, it is recommended that comprehensive assays are employed to elucidate a full profile of antioxidant activity against various reactive oxygen species.

To the best of our knowledge, this is the first research that investigated the

433 antioxidant capacity of extracts from oak wood that can be conferred to aged wines and 434 how the toasting process can influence it. Non-toasted oak wood samples showed more antioxidant power which was very closely correlated with the total polyphenolic content of the samples. The main compounds responsible for the antioxidant capacity of extracts

437 from oak wood were some phenolic acids including gallic, protocatechuic, caffeic and 438 p-coumaric acids and also all the ellagitanins studied.

\section{Acknowledgments}

442 The authors are grateful to the Spanish Ministerio de Ciencia e Innovación for the award 443 of a doctoral grant to MEA and for its financial support towards the Project AGL2008444 04913-CO2-01/ALI.

\section{References}


447 Alonso, A.M., Castro, R., Rodríguez, M.C., Guillén D.A., \& Barroso, C.G. (2004).

448 Study of the antioxidant power of brandies and vinegars derived from Sherry wines and 449 correlation with their content in polyphenols. Food Research International, 2004, 37, $450 \quad 715-721$.

451

452 Awika, J.M., Rooney, L.W., Wu, X., Prior, R.L., \& Cisneros-Zevallos, L. (2003).

453 Screening methods to measure antioxidant activity of sorghum (Sorghum bicolor) and 454 sorghum products. Journal of Agricultural and Food Chemistry, 51, 6657-6662.

Bakkalbase, E., Mentes, Ö., \& Artik, N. (2009). Food ellagitannins-ocurrence, effects of Processing and storage. Critical Reviews in Food Science and Nutrition, 49, 283-298.

Benzie, I.F.F., \& Strain, J.J. (1999). Ferric reducing antioxidant power assay: Direct measure of total antioxidant activity of biological fluids and modified version for simultaneous measurement of total antioxidant power and ascorbic acid concentration. Methods in Enzymology. 1999, 299, 15-27.

463

Brand-Williams, W., Cuvelier, M.E., \& Berset, C. (1995). Use of free radical method to 465 evaluate antioxidant activity. Lebensmittel-Wissenschaft und-Technologie, 28, 25-30.

467 Burns, J., Gardner, P. T., Matthews, D., Duthie, G.G., Lean, M.E.J., \& Crozier, A. 468 (2001). Extraction of phenolics and changes in antioxidant activity of red wines during 469 vinification. Journal of Agricultural and Food Chemistry, 49, 5797-5808.

470 
471 Canas, S., Casanova, V., \& Belchior, A.P. (2008). Antioxidant activity and phenolic

472 content of Portuguese wine aged brandies. Journal of Food Composition and Analysis, $47321,626-633$.

474

475 Cao, G., \& Prior, R. (1999) Measurement of oxygen radical absorbance capacity in 476 biological samples. Oxidants and antioxidants. Methods in Enzymology, 299, 50-62.

477

478 Fernández de Simón. B., Cadahia. E., Conde. E., García-Vallejo. M.C. (1999).

479 Evolution of phenolic compounds of Spanish oak wood during natural seasoning. First 480 results. Journal of Agricultural and Food Chemistry, 47, 1687-1694.

481

482 Fernández-Pachón, M.S., Villaño, D., García-Parilla, M.C., \& Toncoso, A.M. (2004).

483 Antioxidant activity of wines and relation with their polyphenolic composition. 484 Analytica Chimica Acta, 513, 113-118.

485

486 Frankel, E.N., \& Meyer, A. (2000). The problems of using one-dimensional methods to 487 evaluate multifunctional food and biological antioxidants. Journal of Science and Food 488 Agricultural, 80, 1925-1941.

489

490 Fuhrman, D., Volkova, N., Suraski, A., \& Aviram, M. (2001). White wine with red 491 wine-like properties: increased extraction of grape skin polyphenols improves the 492 antioxidant capacity of the derived white wine. Journal of Agricultural and Food 493 Chemistry, 49, 3164-3168. 
Hale, M.D., McCafferty, K., Larmie, E., Newton, J., \& Swan, J.S. (1999). The influence

496 of oak seasoning and toasting parameters on the composition and quality of wine.

497 American Journal of Enology and Vitculture, 50, 495-502.

498

499 Jordão, A.M., Ricardo. J.M., \& Laureano. O. (2007). Ellagitannins from Portuguese oak

500 wood (Quercus pyrenaica Willd.) used in cooperage: influence of geographical origin,

501 coarseness of the grain and toasting level. Holzforschung. 61. 155-160.

502

503 Kaur, C., \& Dapoor, H.C. (2001). Antioxidants in fruits and vegetables - the 504 millenium's health. International Journal of Food Science and Technology, 36, 703505275.

506

507 Larrauri, J.A., Sánchez-Moreno, C., Rupérez, P., \& Saura-Calixto, F. (1999). Free 508 radical scavenging capacity in the aging of selected red Spanish wines. Journal of 509 Agricultural and Food Chemistry, 47, 1603-1606.

510

511 Manzocco, L., Mastrocloa, D., \& Nicoli, M.C. (1999). Chain-breading and oxygen scavenging properties of wine as affected by some technological procedures. Food Research International, 31, 673-678.

515 Ou, B., Huang, D., Hampsch-Woodill, M., Flanagan, J.A., \& Deemer, E.K. (2002).

516 Analysis of antioxidant activities of common vegetables employing oxygen radical

517 absorbance capacity (ORAC) and ferric reducing antioxidant power (FRAP) assays: A

518 Comparative study. Journal of Agricultural and Food Chemistry, 50, 3122-3128. 
520 Pellegrini, N., Simonetti, P., Gardana, C., Brenna, O., Brighenti, F., \& Pietta, P. (2000).

521 Polyphenol content and total antioxidant activity of Vini Novelli (young red wines).

Journal of Agricultural and Food Chemistry, 48, 732-735.

523

524 Pérez-Coello, M.S., Sanchéz, M.A., García, E., González-Viñas, M.A., Sanz, J., \&

525 Cabezudo, M.D. (2000). Fermentation of White wines in the presence of wood chips of

526 American and French oak. Journal of Agricultural and Food Chemistry, 48, 885-889.

527

528 Quideau, S. (2009). An underestimate class of bioactive plant polyphenols. Singapore:

529 World Scientific Publishing Co. Pte. Ltd.

530

531 Re, R., Pellegrini, N., Proteggente, A., Pannala, A., Yang, M., \& Rice-Evans, C. (1999).

532 Antioxidant activity applying an improved ABTS radical cation decolorization assay.

533 Free Radical Biology and Medicine, 26, 1231-1237.

534

535 Record, I.R., Dreosti, I.E., \& Mclnerney, J.K. (2001). Changes in plasma antioxidant

536 status following consumption of diets high or low in fruit and vegetables or following 537 dietary supplementation with an antioxidant mixture. British Journal of Nutrition, 85, 538 459- 464.

539

540 Renaud, S.C., Guegue, R., Schenker, J., \& d’Houtaud, A. (1998). Alcohols and 541 mortality in middle-aged men from eastern France. Epidemiology, 9, 184-188. 
543 Rivero-Pérez, M.D., Gónzalez-Sanjosé, M.L., Muñiz, P., \& Pérez-Magariño, S. (2008).

544 Antioxidant profile of red- single variety wines microoxygenated before malolactic 545 fermentation. Food Chemistry, 111, 1004-1011.

546

547 Singleton, V.L., Rossi, J.A. (1965) Colorimetry of Total Phenolics with

548 Phosphomolybdic - Phosphotungstic Acid Reagents. American Journal of Enology and

549 Viticulture, 16, 144-158.

550

551 Thaipong, K., Boonprakob, U., Crosby, K., Cisneros-Zevallos, L., \& Hawkins-Byrne, 552 D. (2006). Comparison of ABTS, DPPH, FRAP and ORAC assay for estimating 553 antioxidant activity form guava fruit extracts. Journal of Food Composition and 554 Analysis, 19, 669-675.

555

556 Tomera, J.F. (1999). Current knowledge of the health benefits and disadvantages of 557 wine consumption. Trends in Food Science and Technology, 10, 129-138.

558

559 Viriot, C., Scalbert, A., Lapierre, C., \& Moutounet, M. (1993). Ellagitannins and lignins 560 in aging of spirits in oak barrels. Journal of Agricultural and Food Chemistry, 41, 18725611879.

562

563 Viriot. C., Scalbert. A., Herve du Penhoat. C.L. M., \& Moutounet. M. (1994)

564 Ellagitannins in woods of sessile oak and sweet chestnut. Dimerization and hydrolysis 565 during wood aging. Phytochemistry, 36. 1253-1260 
576 Table 1. Total phenol index and antioxidant capacity of oak wood determined by the

\begin{tabular}{lccccc}
\hline \multicolumn{1}{c}{ Oak wood } & $\begin{array}{c}\text { TPI }^{*} \\
\mathrm{n}=4\end{array}$ & $\begin{array}{c}\mathbf{D P P H}^{\boldsymbol{\delta}} \\
\mathrm{n}=4\end{array}$ & $\begin{array}{c}\mathbf{F R A P}^{\boldsymbol{\delta}} \\
\mathrm{n}=4\end{array}$ & $\begin{array}{c}\mathbf{O R A C}^{\boldsymbol{\delta}} \\
\mathrm{n}=4\end{array}$ & $\begin{array}{c}\mathbf{A B T S}^{\mathbf{\delta}} \\
\mathrm{n}=4\end{array}$ \\
\hline American & $32.28^{\mathrm{b}} \pm 0.45$ & $0.26^{\mathrm{b}} \pm 0.02$ & $0.29^{\mathrm{b}} \pm 0.02$ & $0.42^{\mathrm{a}} \pm 0.01$ & $0.55^{\mathrm{b}} \pm 0.01$ \\
American toasted & $22.52^{\mathrm{a}} \pm 0.95$ & $0.17^{\mathrm{a}} \pm 0.01$ & $0.18^{\mathrm{a}} \pm 0.01$ & $0.38^{\mathrm{a}} \pm 0.02$ & $0.39^{\mathrm{a}} \pm 0.01$ \\
\hline French & $50.95^{\mathrm{b}} \pm 1.58$ & $0.45^{\mathrm{b}} \pm 0.05$ & $0.45^{\mathrm{b}} \pm 0.03$ & $0.46^{\mathrm{a}} \pm 0.02$ & $0.97^{\mathrm{b}} \pm 0.06$ \\
French toasted & $29.52^{\mathrm{a}} \pm 0.48$ & $0.23^{\mathrm{a}} \pm 0.02$ & $0.32^{\mathrm{a}} \pm 0.05$ & $0.41^{\mathrm{a}} \pm 0.02$ & $0.74^{\mathrm{a}} \pm 0.08$ \\
\hline Hungarian & $45.72^{\mathrm{b}} \pm 0.95$ & $0.35^{\mathrm{b}} \pm 0.06$ & $0.44^{\mathrm{b}} \pm 0.03$ & $0.44^{\mathrm{a}} \pm 0.02$ & $0.94^{\mathrm{b}} \pm 0.02$ \\
Hungarian toasted & $22.90^{\mathrm{a}} \pm 1.28$ & $0.14^{\mathrm{a}} \pm 0.02$ & $0.15^{\mathrm{a}} \pm 0.03$ & $0.37^{\mathrm{a}} \pm 0.01$ & $0.49^{\mathrm{a}} \pm 0.05$ \\
\hline Rumanian & $25.30^{\mathrm{b}} \pm 0.44$ & $0.18^{\mathrm{b}} \pm 0.03$ & $0.20^{\mathrm{b}} \pm 0.01$ & $0.39^{\mathrm{a}} \pm 0.04$ & $0.44^{\mathrm{b}} \pm 0.02$ \\
Rumanian toasted & $14.37^{\mathrm{a}} \pm 0.97$ & $0.08^{\mathrm{a}} \pm 0.01$ & $0.10^{\mathrm{a}} \pm 0.01$ & $0.32^{\mathrm{a}} \pm 0.03$ & $0.29^{\mathrm{a}} \pm 0.02$ \\
\hline Russian & $25.95^{\mathrm{b}} \pm 0.76$ & $0.21^{\mathrm{b}} \pm 0.02$ & $0.20^{\mathrm{b}} \pm 0.01$ & $0.39^{\mathrm{a}} \pm 0.02$ & $0.43^{\mathrm{b}} \pm 0.02$ \\
Russian toasted & $14.41^{\mathrm{a}} \pm 0.39$ & $0.08^{\mathrm{a}} \pm 0.01$ & $0.08^{\mathrm{a}} \pm 0.00$ & $0.33^{\mathrm{a}} \pm 0.03$ & $0.25^{\mathrm{a}} \pm 0.00$ \\
\hline
\end{tabular}

"TPI: Total phenol index expressed as micrograms of gallic acid equivalents per

580 milligram of oak wood

$581{ }^{ð}$ Expressed as $\mu \mathrm{mol}$ of Trolox equivalents per milligram of oak wood

582 Different superscript letters in the same column denote a significant difference among

583 non-toasted and toasted samples according to the Student-Newman-Keuls test at $\mathrm{P}<$

$584 \quad 0.05$ 
594 Table 2. Pearson's coefficient between different antioxidant capacity methods tested 595 and the total phenol index.

596

\begin{tabular}{ccccc}
\hline & DPPH & FRAP & ORAC & ABTS \\
\hline TPI & $0.97^{*}$ & $0.96^{*}$ & $0.72^{*}$ & $0.95^{*}$ \\
\hline DPPH & & $0.92^{*}$ & $0.69^{*}$ & $0.90^{*}$ \\
\hline FRAP & & $0.73^{*}$ & $0.95^{*}$ \\
\hline ORAC & & & & $0.74^{*}$ \\
\hline
\end{tabular}

597

$598 *$ Significant correlation $\mathrm{p}<0.01$ (bilateral)

599 
Table 3. Concentrations of low molecular weight phenolic compounds expressed as $\mu \mathrm{g} \mathrm{g}^{-1}$ in toasted and non-toasted oak wood samples ( $\mathrm{n}=2$ )

\begin{tabular}{lccccccccccc}
\hline \multirow{2}{*}{ Compound } & \multicolumn{2}{c}{ American oak wood } & \multicolumn{2}{c}{ French oak wood } & \multicolumn{2}{c}{ Hungarian oak wood } & \multicolumn{2}{c}{ Rumanian oak wood } \\
\cline { 2 - 8 } & Natural & Toasted & Natural & Toasted & Natural & Toasted & Natural & Toasted & Natural & Toasted \\
\hline Gallic acid & $396.1 \pm 10.6$ & $249.7 \pm 6.1$ & $442.8 \pm 5.4$ & $343.5 \pm 8.0$ & $392.8 \pm 6.6$ & $146.3 \pm 7.7$ & $220.2^{*} \pm 0.7$ & $195.5^{*} \pm 10.9$ & $149.4 \pm 2.0$ & $110.0 \pm 6.0$ \\
Protocatechuic acid & $221.5 \pm 4.7$ & $81.7 \pm 8.7$ & $256.0 \pm 9.3$ & $130.2 \pm 9.6$ & $242.8 \pm 12.3$ & $82.9 \pm 4.0$ & $231.5 \pm 11.4$ & $66.1 \pm 5.6$ & $135.1 \pm 8.0$ & $23.9 \pm 1.5$ \\
Protocatechuic aldehyde & nd & $112.3 \pm 2.0$ & nd & $116.0 \pm 5.9$ & $9.9 \pm 1.8$ & $32.4 \pm 4.2$ & $n d$ & $11.2 \pm 0.6$ & $n d$ & $34.8 \pm 4.7$ \\
Vanillic acid & $91.6 \pm 8.5$ & $129.9 \pm 3.2$ & $87.1 \pm 14.8$ & $140.2 \pm 5.2$ & $70.8 \pm 0.7$ & $96.8 \pm 1.9$ & $45.7^{*} \pm 1.0$ & $43.2^{*} \pm 0.3$ & $39.6 \pm 2.7$ & $70.6 \pm 9.4$ \\
Caffeic acid & $101.0 \pm 2.6$ & $26.4 \pm 0.9$ & $89.5 \pm 1.1$ & $18.3 \pm 1.1$ & $36.6 \pm 2.0$ & $4.6 \pm 0.5$ & $46.9 \pm 2.1$ & $6.1 \pm 0.5$ & $46.0 \pm 0.9$ & $7.5 \pm 0.5$ \\
Vanillin & $106.4 \pm 10.8$ & $210.2 \pm 3.7$ & $94.7 \pm 10.2$ & $204.0 \pm 10.1$ & $76.7 \pm 7.7$ & $192.0 \pm 0.1$ & $45.6 \pm 2.7$ & $133.5 \pm 1.1$ & $37.0 \pm 1.8$ & $208.9 \pm 9.9$ \\
Syringaldehyde & $97.9 \pm 7.3$ & $523.8 \pm 4.2$ & $41.8 \pm 0.5$ & $597.4 \pm 7.5$ & $57.6 \pm 1.7$ & $633.8 \pm 8.3$ & $19.7 \pm 0.2$ & $471.6 \pm 14.0$ & $55.5 \pm 0.6$ & $657.5 \pm 10.4$ \\
$p$-coumaric acid & $27.5^{*} \pm 1.0$ & $20.4^{*} \pm 1.8$ & $31.5^{*} \pm 0.7$ & $25.9 * \pm 3.3$ & $32.7 * \pm 2.5$ & $26.5^{*} \pm 0.4$ & $18.8^{*} \pm 0.3$ & $16.2^{*} \pm 0.7$ & $20.5 \pm 0.8$ & $15.6 \pm 0.6$ \\
Scopoletin & $54.6 \pm 2.5$ & $35.0 \pm 2.0$ & $25.0^{*} \pm 3.0$ & $21.1^{*} \pm 0.5$ & $27.5 \pm 1.5$ & $18.2 \pm 1.5$ & $22.9 \pm 1.0$ & $15.4 \pm 1.5$ & $30.4 \pm 2.5$ & $16.1 \pm 1.5$ \\
Ferulic acid & $59.0 \pm 3.6$ & $85.9 \pm 3.0$ & $54.1 \pm 0.6$ & $74.2 \pm 4.8$ & $24.6^{*} \pm 1.5$ & $29.9 * \pm 0.9$ & $20.5 \pm 1.0$ & $28.0 \pm 1.2$ & $20.4 \pm 1.5$ & $32.0 \pm 2.2$ \\
Sinapic acid & $41.2 \pm 2.8$ & $61.1 \pm 3.5$ & $25.1 \pm 1.0$ & $53.9 \pm 3.5$ & $17.5 \pm 3.5$ & $46.5 \pm 3.7$ & $26.2 \pm 1.1$ & $48.6 \pm 3.0$ & $10.4 \pm 0.5$ & $39.1 \pm 3.8$ \\
Coniferaldehyde & $111.2 \pm 9.3$ & $352.1 \pm 9.1$ & $112.5 \pm 12.2$ & $298.7 \pm 2.4$ & $17.3 \pm 3.9$ & $198.3 \pm 11.5$ & $50.3 \pm 0.8$ & $221.9 \pm 8.6$ & $39.4 \pm 0.7$ & $220.8 \pm 12.4$ \\
Sinapaldehyde & $261.2 \pm 9.6$ & $1112.2 \pm 19.4$ & $305.7 \pm 8.5$ & $1043.0 \pm 15.7$ & $68.4 \pm 3.1$ & $605.7 \pm 13.4$ & $154.6 \pm 3.1$ & $590.0 \pm 8.7$ & $87.3 \pm 12.4$ & $780.5 \pm 11.9$ \\
Ellagic acid & $199.1 \pm 8.7$ & $236.0 \pm 3.55$ & $206.6 \pm 9.1$ & $267.0 \pm 8.0$ & $126.1 \pm 6.9$ & $199.3 \pm 6.1$ & $85.8 \pm 6.1$ & $247.5 \pm 4.5$ & $99.4 \pm 3.7$ & $154.8 \pm 4.4$ \\
\hline
\end{tabular}

$602 *$ No differences were found between non-toasted and respectively toasted samples $\mathrm{p}<0.05$

603 Table 4. Concentrations of ellagitannins (expressed as $\mu \mathrm{g} \mathrm{g}^{-1}$ ellagic acid equivalents) in toasted and non-toasted oak wood samples ( $\mathrm{n}=2$ ). 


\begin{tabular}{lcccccccccc}
\hline \multirow{2}{*}{ Compound } & \multicolumn{2}{c}{ American oak wood } & \multicolumn{2}{c}{ French oak wood } & \multicolumn{2}{c}{ Hungarian oak wood } & \multicolumn{2}{c}{ Rumanian oak wood } & Russian oak wood \\
\cline { 2 - 9 } & Natural & Toasted & Natural & Toasted & Natural & Toasted & Natural & Toasted & Natural & Toasted \\
\hline Roburin A & $60.4 \pm 3.1$ & $26.3 \pm 0.9$ & $155.9 \pm 3.2$ & $72.3 \pm 0.7$ & $85.1 \pm 1.7$ & $21.9 \pm 0.5$ & $72.3 \pm 1.3$ & $0.1 \pm 0.0$ & $88.9 \pm 2.1$ & $0.1 \pm 0.0$ \\
& & & & & & & & & & \\
Roburin B & $77.4 \pm 5.0$ & $35.1 \pm 1.4$ & $118.5 \pm 1.3$ & $49.0 \pm 2.3$ & $105.1 \pm 1.4$ & $26.6 \pm 1.1$ & $77.2 \pm 1.0$ & $0.1 \pm 0.0$ & $88.3 \pm 0.8$ & $0.1 \pm 0.0$ \\
Roburin C & $87.0 \pm 4.7$ & $36.0 \pm 0.9$ & $156.7 \pm 0.5$ & $68.8 \pm 1.3$ & $108.4 \pm 1.7$ & $29.0 \pm 1.5$ & $94.5 \pm 2.7$ & $0.1 \pm 0.0$ & $92.8 \pm 3.0$ & $0.1 \pm 0.0$ \\
Grandinin & $242.5 \pm 4.2$ & $60.1 \pm 0.9$ & $325.1 \pm 15.7$ & $93.9 \pm 0.5$ & $439.8 \pm 16.9$ & $49.8 \pm 1.2$ & $260.2 \pm 0.4$ & $19.2 \pm 2.2$ & $260.1 \pm 6.7$ & $15.6 \pm 1.4$ \\
Roburin D & $44.7 \pm 1.9$ & $15.0 \pm 0.9$ & $188.0 \pm 0.7$ & $63.9 \pm 1.63$ & $70.4 \pm 3.2$ & $11.3 \pm 0.2$ & $55.6 \pm 0.6$ & $0.1 \pm 0.0$ & $60.2 \pm 2.0$ & $0.1 \pm 0.0$ \\
Vescalagin & $349.8 \pm 3.2$ & $106.9 \pm 0.1$ & $1076.6 \pm 6.4$ & $293.6 \pm 4.2$ & $721.9 \pm 5.5$ & $150.2 \pm 9.6$ & $376.0 \pm 14.1$ & $43.5 \pm 3.5$ & $430.4 \pm 16.1$ & $40.8 \pm 2.3$ \\
Roburin E & $472.8 \pm 9.9$ & $130.4 \pm 8.5$ & $949.1 \pm 9.5$ & $248.2 \pm 8.0$ & $751.6 \pm 21.5$ & $76.7 \pm 1.7$ & $515.9 \pm 3.8$ & $42.6 \pm 4.3$ & $488.7 \pm 20.2$ & $35.6 \pm 2.3$ \\
Castalagin & $724.4 \pm 6.9$ & $316.0 \pm 5.2$ & $1995.0 \pm 128.7$ & $902.7 \pm 18.0$ & $1642.3 \pm 23.2$ & $455.2 \pm 9.4$ & $790.8 \pm 11.1$ & $223.1 \pm 9.5$ & $734.9 \pm 49.9$ & $131.2 \pm 6.4$ \\
\hline
\end{tabular}


607 Table 5. Correlation matrix between antioxidant capacity methods tested and the

608 concentration of each phenolic compound.

609

\begin{tabular}{lcccc}
\hline \multicolumn{1}{c}{ Compound } & DPPH & FRAP & ORAC & ABTS \\
\hline Gallic acid & $0.85^{*}$ & $0.90^{*}$ & $0.83^{*}$ & $0.84^{*}$ \\
Protocatechuic acid & $0.84^{*}$ & $0.82^{*}$ & $0.84^{*}$ & $0.71^{*}$ \\
Protocatechuic aldehyde & -0.26 & -0.12 & -0.08 & -0.06 \\
Vanillic acid & 0.14 & 0.24 & 0.31 & 0.29 \\
Caffeic acid & $0.69^{*}$ & $0.62^{*}$ & $0.73^{*}$ & 0.47 \\
Vanillin & -0.47 & -0.38 & -0.38 & -0.27 \\
Syringaldehyde & $-0.68^{*}$ & $-0.59^{*}$ & $-0.62^{*}$ & -0.45 \\
p-coumaric acid & $0.84^{*}$ & 0.87 & $0.81^{*}$ & $0.91^{*}$ \\
Scopoletin & 0.32 & 0.33 & 0.44 & 0.14 \\
Ferulic acid & 0.14 & 0.23 & 0.32 & 0.18 \\
Sinapic acid & -0.50 & -0.38 & -0.34 & -0.33 \\
Coniferaldehyde & -0.49 & -0.41 & -0.37 & -0.34 \\
Sinapaldehyde & -0.48 & -0.38 & -0.35 & -0.31 \\
Ellagic acid & -0.12 & 0.02 & -0.09 & 0.04 \\
Roburin A & $0.90^{*}$ & $0.84^{*}$ & $0.87^{*}$ & $0.81^{*}$ \\
Roburin B & $0.90^{*}$ & $0.85^{*}$ & $0.88^{*}$ & $0.77^{*}$ \\
Roburin C & $0.92^{*}$ & $0.86^{*}$ & $0.91^{*}$ & $0.80^{*}$ \\
Grandinin & $0.85^{*}$ & $0.80^{*}$ & $0.76^{*}$ & $0.72^{*}$ \\
Roburin D & $0.89^{*}$ & $0.82^{*}$ & $0.83^{*}$ & $0.81^{*}$ \\
Vescalagin & $0.96^{*}$ & $0.88^{*}$ & $0.84^{*}$ & $0.86^{*}$ \\
Roburin E & $0.93^{*}$ & $0.86^{*}$ & $0.85^{*}$ & $0.80^{*}$ \\
Castalagin & $0.97^{*}$ & $0.94^{*}$ & $0.86^{*}$ & $0.94^{*}$ \\
\hline
\end{tabular}

610

$611 *$ Significant correlation at the 0.01 level (two-tailed). 